\title{
Regorafenib-induced hypothyroidism and cancer-related fatigue: is there a potential link?
}

\author{
Fabiana Pani', Matteo Massidda1, Valeria Pusceddu², Marco Puzzoni², \\ Elena Massa' ${ }^{2}$, Clelia Madeddu ${ }^{2}$, Mario Scartozzi ${ }^{2}$ and Stefano Mariotti ${ }^{1}$ \\ ${ }^{1}$ Endocrinology Unit and ${ }^{2}$ Medical Oncology Unit, Department of Medical Sciences and Public Health, University of \\ Cagliari, Cagliari, Italy
}

Correspondence should be addressed to F Pani

Email

panifabiana@gmail.com

\begin{abstract}
Objective: Thyroid dysfunction has been reported during Regorafenib (Reg) administration, but no detailed study is presently available.

Design: Prospective, observational cohort study. Patients with documented metastatic colorectal cancer and progression of disease during or within 3 months after the last standard therapy, with no evidence and history of previous thyroid disease were enrolled.

Methods: Twenty-five consecutive patients were evaluated before and 8-50 weeks after initiating Reg therapy by monthly clinical, ultrasound and laboratory (thyrotropin (TSH), free thyroxine (fT4), antithyroglobulin (TgAb) and antithyroid peroxidase (TPOAb)) evaluation.

Results: Thirteen/25 patients (52\%) became hypothyroid (TSH: $12.5 \pm 4.01 \mathrm{IU} / \mathrm{L}$, range: $4.6-22.0$ ) within 5 months of therapy. TPOAb became detectable $(99-155 \mathrm{IU} / \mathrm{mL}$ ) in $2 / 25(8 \%)$ patients. Thyroid volume progressively decreased (from $8.6 \pm 2.2 \mathrm{~mL}$ to $4.9 \pm 2.4 \mathrm{~mL}$ after 5 months of Reg therapy, $P<0.0001$ ). The progression-free survival (PFS) was longer in patients developing hypothyroidism (43 weeks) than in those remaining euthyroid (17 weeks, $P<0.01$ ). Fatigue (the most common general serious Reg adverse event) was associated with hypothyroidism severity and reversed after levothyroxine therapy (L-T4).

Conclusions: Reg rapidly causes hypothyroidism in about $50 \%$ of patients and in a minority of them also triggers thyroid autoimmunity. Reg-induced hypothyroidism was strictly related to fatigue, easily reversed by L-T4 administration and associated to longer survival. These results suggest that prompt recognition of hypothyroidism in patients with severe fatigue may prevent unnecessary Reg dose reduction or withdrawal.
\end{abstract}

\section{Introduction}

Regorafenib (Reg) is a novel oral multi-tyrosine kinase inhibitor (TKI) recently approved for the treatment of progressive metastatic colorectal carcinoma (mCRC) (1) after failure of all other approved standard therapy (at least three lines) including chemotherapy-based on fluoropyrimidines, oxaliplatin, irinotecan, monoclonal antibodies-targeting vascular endothelial growth factor (VEGF) and an anti-epidermal growth factor receptor (EGFR) in KRAS wild-type patients (2).
() 2017 European Society of Endocrinology Printed in Great Britain
Reg is able to block various signaling pathways implicated in the development of mCRC by inhibition on the activity of specific proteins involved in regulation of tumor angiogenesis (VEGFR1-3), oncogenesis (KIT, RET, RAF1 and BRAF) and microenvironment (PDGFR and FGFR) (2, 3). Compared with conventional cytotoxic chemotherapy agents, this drug is easier to administer; however, the toxicity profile includes several adverse events (AEs) such as fatigue, hand foot skin reaction

Published by Bioscientifica Ltd. 
(HFS), hypertension, diarrhea, liver dysfunction and thyroid dysfunction $(3,4,5)$. Cancer-related fatigue (CRF) is the most frequent and earlier $\mathrm{AE}$ of Reg: its precise etiology is difficult to identify and to manage for the advanced stage of the primary tumor and the frequent presence of several comorbidities (anemia, cachexia, depression and others) $(6,7)$. Thyroid dysfunctions (either hypothyroidism or hyperthyroidism) have been occasionally described on Reg therapy $(3,5,8)$ and could be implicated in the development of CRF. However, at difference with other TKI (sunitinib, sorafenib, axitinib, dasatinib, cabozantinib and vandetanib) (9), the spectrum, the time of appearance and the potential mechanisms involved in Reg-induced thyroid dysfunctions are still poorly known.

Aim of the present investigation was therefore to longitudinally evaluate thyroid function, thyroid autoimmunity and morphology, before and during Reg administration in a homogenous series of patients with mCRC, with normal thyroid function and no clinical, ultrasound and serological evidence of pre-existing thyroid dysfunction/autoimmunity before starting Reg.

\section{Subjects and methods}

\section{Patients}

A total of 25 consecutive patients (12 males and 13 females) aged $49-77$ years $(64.1 \pm 7.9$ years, $M+$ S.D. $)$ with mCRC currently followed at the Medical Oncology Unit of Cagliari University Hospital were enrolled in the study. All patients had received locally and currently approved standard chemotherapy or had stopped them because of unacceptable AEs. The median number of previous palliative systemic chemotherapies was 3 . All patients were at a comparable advanced tumor stage with the following distribution of distant metastases: 3/25 (12\%) lung, $10 / 25$ (40\%) bone and 21/25 (84\%) liver; in 68\% of cases, metastatic sites were more than 2. Quality of life (QoL) was assessed with Eastern Cooperative Oncology Group Performance Status (ECOG-PS) (10). The score calculated was between 0 and 5: the lowest score $(0-2)$ identified a patient fully active, which was able to carry out more than $50 \%$ of daily activities; however, a score greater than 2 identified the patients limited or completely disabled in their daily activities. Thyroid evaluation was performed at the outpatient clinic of the Endocrinology Unit of Cagliari University Hospital. All patients were euthyroid with no serological and/or ultrasound evidence of associated thyroid autoimmunity and a negative familial history of thyroid disease. The full inclusion and exclusion criteria are detailed in Table 1.

All patients were initially treated with Reg (Stivarga) for $8-50$ weeks $(4.9 \pm 2.2$ months, mean \pm s.D. $)$. Reg was given at a daily oral dose of $160 \mathrm{mg}$ for three weeks $(\mathrm{ON})$, followed by one week of withdrawal (OFF) on the basis of the clinical condition. In some cases, Reg was temporarily discontinued, and six patients required dose reductions. Moreover, Reg was permanently withdrawn in 12 patients after $4-5$ months for progressive disease.

Table 1 Summary of eligibility criteria.

Inclusion criteria
Signed informed consent: patients must be able to sign it
Male or female patients: $\geq 18$ years of age
Histological or cytological documentation of adenocarcinoma
of the colon or rectum
Progression during or within 3 months following the last
administration of approved fewer than three standard
therapies
Measurable disease according to Response Evaluation Criteria
in Solid Tumors, version 1.1
Eastern Cooperative Oncology Group performance status of
$\leq 2$
Life expectancy of at least 3 months and adequate liver and
renal function
No familial history of thyroid disease

No previous biochemical diagnosis of thyroid dysfunction, thyroid autoimmunity and or/ultrasound evidence of thyroid autoimmunity

\section{Exclusion criteria}

Prior treatment with Regorafenib

Major surgical procedure or significant traumatic injury within

28 days before start of study medication

Eastern Cooperative Oncology Group performance status of $\geq 2$

Concomitant congestive heart failure of New York Association class 2 or worse

Concomitant unstable angina and myocardial infarction less than 6 months before start of study group

Uncontrolled hypertension

Family history of thyroid disease

Previous diagnosis of biochemical thyroid dysfunction, thyroid autoimmunity and/or ultrasound evidence of pattern like-autoimmunity 
Tumor measurements were evaluated by the treating oncologist according to the Response Evaluation Criteria in Solid Tumors (RECIST) (11). Computed tomography scan or pelvic magnetic resonance imaging was performed every 12 weeks during Reg treatment.

Thyroid dysfunction was defined according to international guidelines (12).

All patients signed an informed consent, and the study was approved by the Institutional Local Ethics Committee.

\section{Biochemical and instrumental investigations}

Blood samples were collected before and at approximately monthly intervals either in 'ON' and 'OFF' periods during the Reg administration. Thyrotropin (TSH; reference range: 0.4-4.0 IU/L), free thyroxine (fT4; $0.9-1.9 \mathrm{ng} / \mathrm{dL}$ ), free antithyroglobulin (TgAb; 20-40 IU/mL) and antithyroid peroxidase (TPOAb; 10-35 IU/mL) autoantibodies were measured by an ultrasensitive chemiluminescent assay (Immulite $2000 \mathrm{XPi}$; Siemens Milan). All patients underwent a careful clinical examination, followed by thyroid ultrasound using echo-color Doppler technique, always performed by the same operator (F.P.) and with the same instrument (Sonoline G60S; Siemens Medical Solutions). In all cases, the estimated thyroid volume (ETV) was evaluated using the formula of the ellipsoid of rotation (depth·width·length·0.52) (13). The parenchymal echogenicity and the presence of thyroid nodules were always assessed.

\section{Statistical analysis}

Parametric (Student's t-test), nonparametric (MannWhitney $U$-test) tests and linear correlation were used to compare the two groups of patients. The hazard ratio (HR) and confidence interval (CI) were estimated by stratified Cox proportional hazards regression. Statistical significance was considered at $P<0.05$. All calculations were performed using GraphPad Prism.

\section{Results}

\section{Patients characteristics}

Sex, age, basal clinical assessment by ECOG-PS and length of follow-up in the 25 patients studied are summarized in Table 2, where the indication of thyroid dysfunction in individual cases is also shown. Before Reg administration,
Table 2 Age, sex and basal clinical assessment by ECOG-PS in 25 patients. The length of follow-up and the individual development of hypothyroidism (Hypo) and/or TPOAb are also shown.

\begin{tabular}{|c|c|c|c|c|}
\hline Patient \# & Age/sex & ECOG-PS & Follow-up (weeks) & Нуро-ТРОАЬ \\
\hline 1 & $76 / \mathrm{F}$ & 2 & 12 & No \\
\hline 2 & $61 / \mathrm{M}$ & 1 & 13 & No \\
\hline 3 & $74 / F$ & 1 & 15 & No \\
\hline 4 & $54 / \mathrm{M}$ & 1 & 20 & Yes/No \\
\hline 5 & $66 / F$ & 1 & 24 & Yes/No \\
\hline 6 & 63/M & 1 & 24 & No \\
\hline 7 & $58 / F$ & 1 & 26 & Yes/No \\
\hline 8 & 66/M & 1 & 50 & Yes/Yes \\
\hline 9 & $66 / F$ & 2 & 20 & Yes/No \\
\hline 10 & 49/F & 2 & 21 & Yes/No \\
\hline 11 & 69/F & 2 & 11 & No \\
\hline 12 & 61/F & 1 & 43 & Yes/Yes \\
\hline 13 & 68/M & 1 & 20 & No \\
\hline 14 & $57 / F$ & 1 & 21 & No \\
\hline 15 & $62 / F$ & 2 & 14 & No \\
\hline 16 & $64 / M$ & 1 & 27 & Yes/No \\
\hline 17 & $75 / F$ & 1 & 25 & Yes/No \\
\hline 18 & 77/M & 2 & 21 & No \\
\hline 19 & 74/M & 2 & 8 & No \\
\hline 20 & 75/M & 1 & 13 & No \\
\hline 21 & $58 / \mathrm{M}$ & 1 & 19 & Yes/No \\
\hline 22 & $58 / F$ & 1 & 16 & No \\
\hline 23 & $58 / \mathrm{M}$ & 1 & 23 & Yes/No \\
\hline 24 & $58 / \mathrm{M}$ & 1 & 17 & No \\
\hline 25 & $58 / F$ & 2 & 9 & No \\
\hline
\end{tabular}

all patients had normal TSH with TgAb and TPOAb within the reference range $(20-40 \mathrm{IU} / \mathrm{mL}$ and $10-35 \mathrm{IU} / \mathrm{mL}$ respectively). All patients had received fewer than 3 standard chemotherapies. The median duration of Reg treatment was 20 weeks (range: 8-50 weeks).

\section{Thyroid function during Reg administration}

During the first five months of therapy, increased serum TSH concentrations were often observed in patients during the $\mathrm{ON}$ period, followed by normalization in the OFF period. According to international guidelines (12), subclinical primary hypothyroidism was diagnosed in all patients with serum TSH concentration $>4.5 \mathrm{IU} / \mathrm{L}$, with fT4 within the normal range and clinical hypothyroidism in patients with increased TSH and decreased fT4. One patient developed hypothyroidism after early transient period of suppressed TSH $(<0.01 \mathrm{IU} / \mathrm{L})$, presumably due to mild destructive thyrotoxicosis. Serum fT4 displayed a non-significant trend to decrease (data not shown).

Levothyroxine (L-T4) therapy (25-75 $\mu \mathrm{g} /$ day) was started in 11 hypothyroid patients with persistent serum TSH elevation $\geq 10 \mathrm{IU} / \mathrm{L}$ at the end of the 'OFF' 
Table 3 Age, sex, thyroid parameters and grading of fatigue in the 11 patients who developed hypothyroidism.

\begin{tabular}{|c|c|c|c|}
\hline \multirow[b]{2}{*}{ Patient \# } & \multirow[b]{2}{*}{ Age/sex } & \multicolumn{2}{|c|}{$\mathbf{T S H}^{\alpha}(0.4-4.0 \mu \mathrm{IU} / \mathrm{mL})$} \\
\hline & & Before & Last \\
\hline 4 & 54/M & 9.1 & 1.58 \\
\hline 5 & $66 / F$ & 11.2 & 3.8 \\
\hline 7 & $58 / \mathrm{F}$ & 11.8 & 3.5 \\
\hline 8 & $66 / M$ & 12.1 & 2.7 \\
\hline 9 & $66 / F$ & 11.1 & 3.1 \\
\hline 10 & $49 / F$ & 7.9 & 1.58 \\
\hline 12 & $61 / F$ & 15.6 & 1.5 \\
\hline 16 & $64 / M$ & 22.0 & 4.0 \\
\hline 17 & $75 / F$ & 17.9 & 4.0 \\
\hline 21 & 58/M & 9.1 & 2.2 \\
\hline 23 & 58/M & 9.9 & 2.1 \\
\hline
\end{tabular}

\begin{tabular}{ccc}
\hline \multicolumn{2}{c}{ FT4* $^{*}(0.8-1.9 \mathrm{ng} / \mathrm{dL})$} \\
\cline { 1 - 1 } Before & Last \\
\cline { 1 - 1 } 1.41 & & 1.45 \\
1.01 & & 1.39 \\
1.01 & & 1.21 \\
1.26 & & 1.25 \\
0.96 & & 1.10 \\
1.00 & & 1.35 \\
1.03 & & 1.46 \\
0.64 & & 0.96 \\
1.39 & & 1.41 \\
1.24 & 1.09 \\
1.10 & 1.11 \\
\hline
\end{tabular}

\begin{tabular}{|c|c|}
\hline \multicolumn{2}{|c|}{ Grading of fatigue ${ }^{\beta}$} \\
\hline Before & Last \\
\hline 3 & 1 \\
\hline 2 & 1 \\
\hline 2 & 1 \\
\hline 3 & 1 \\
\hline 2 & 1 \\
\hline 3 & 2 \\
\hline 3 & 1 \\
\hline 3 & 1 \\
\hline 3 & 2 \\
\hline 2 & 1 \\
\hline 3 & 1 \\
\hline
\end{tabular}

"The first value represents the highest TSH concentration observed before the beginning of L-T4 therapy and the second value is the last measurement available; *The first value represents the value of fT4 before L-T4 therapy and the second value is the last available after L-T4 treatment; ${ }^{B}$ The first value represents the score of fatigue before L-T4 therapy and the second value is the last after L-T4 treatment.

period (Table 3 for details on individual cases). L-T4 was also started in patients who developed milder forms of hypothyroidism (two consecutive measurements of TSH between 7 and 10IU/L), associated with clinical signs and symptoms highly suggestive of thyroid failure (13). In all L-T4-treated patients, serum TSH was stably maintained within the normal range during all the subsequent time of follow-up.

\section{Antithyroid antibodies during Reg administration}

Although at baseline, both $\mathrm{TgAb}$ and TPOAb were within the reference range $(<20 \mathrm{IU} / \mathrm{mL}$ and $<35 \mathrm{IU} / \mathrm{mL}$ respectively), two (8\%) patients developed mild elevation of TPOAb $(99,155 \mathrm{IU} / \mathrm{mL})$ within 3 months after starting Reg therapy. TPOAb persisted elevated for all the follow-up length (10-12 months). In contrast with TPOAb, TgAb remained consistently undetectable $(<20 \mathrm{IU} / \mathrm{mL})$ during all cycles of treatment (data not shown).

\section{Thyroid function, antithyroid antibodies and tumor response}

As shown in Fig. 1, the progression-free survival (PFS) was longer $(P<0.01$ by Cox regression analyses) in patients with significant hypothyroidism (those requiring L-T4 treatment) as compared to euthyroid patients and those with milder hypothyroidism.

\section{Echo-color Doppler ultrasonography evaluation}

At baseline, all patients showed a normal estimated thyroid volume (ETV) with normal echogenicity, although five of them (patients 3, 4, 12, 16 and 17) displayed one or more thyroid nodules ranging from 12 to $33 \mathrm{~mm}$ in maximal diameter. The nodules observed in patients 4, 12, 16 and 17 were not suspicious at ultrasound examination and the patients refused fine-needle aspiration cytology (FNAC). On the contrary, the nodule observed in patient 3 , which presented some suspicious features of malignancy, was submitted to FNAC that confirmed its malignant nature. The means \pm S.D. and the individual variations of the ETV in all patients are reported in Fig. 2. The mean basal ETV $(8.6 \pm 2.2 \mathrm{~mL})$ displayed a significant reduction already

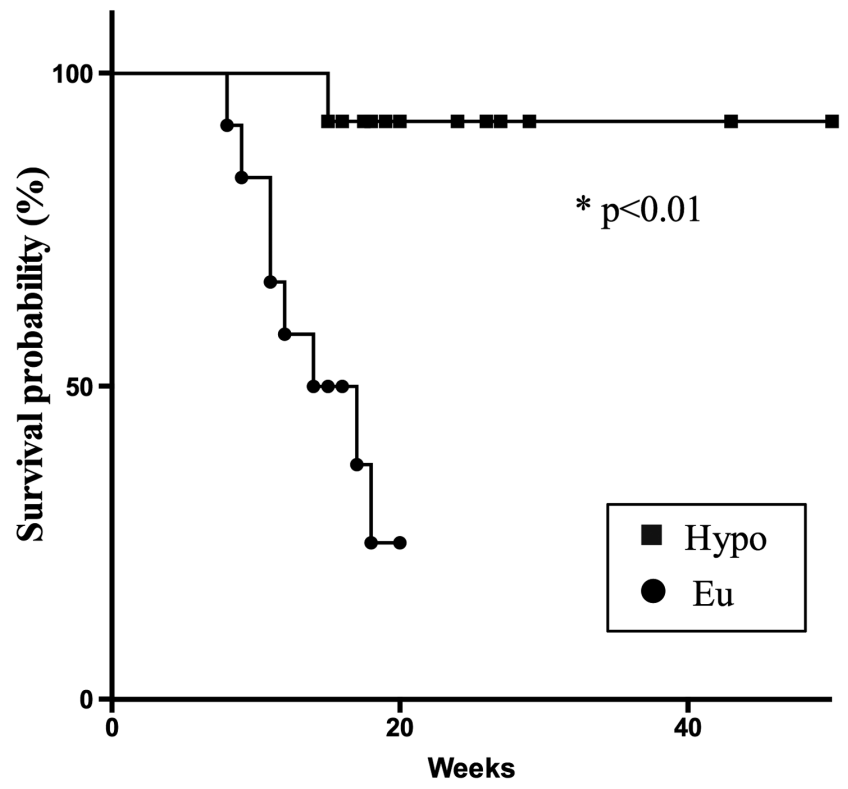

Figure 1

Kaplan-Meier estimate of survival probability in 13 patients who developed hypothyroidism and in 12 patients who remained euthyroid during Reg therapy. 


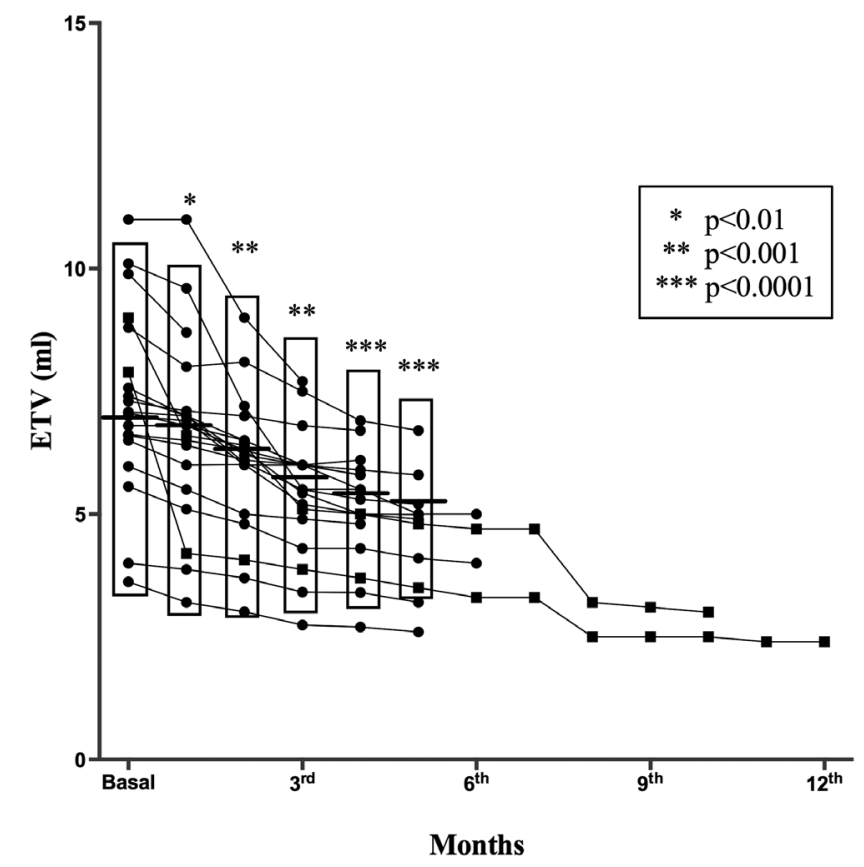

Figure 2

Individual values of estimated thyroid volume (ETV) in 25 patients with MCRC before (basal) and during 2-12 months of Reg treatment; mean \pm S.D. values of ETV up to 5 months are also shown. Solid circles indicate TPOAb-negative and solid squares indicate TPOAb-positive patients.

after one month of treatment $(7.0 \pm 4.2 \mathrm{~mL})$ and then progressively decreased to $4.9 \pm 2.4 \mathrm{~mL}$ after 5 months of treatment $(P<0.0001$ by Mann-Whitney $t$-test). The highest individual percentual reduction $(68 \%$ and $73 \%$ respectively) was observed in the two TPOAb-positive patients, although this phenomenon could be accounted by the longer survival with prolonged exposure to Reg toxicity effect.

Besides the reduction in the ETV, Reg treatment was associated with a change in parenchymal ultrasound features and vascularization characterized by moderate hypoechogenicity more evident in TPOAb-positive patients and by a trend to a reduced parenchymal perfusion, which did not reach the level of statistical significance. Interestingly, in five patients with thyroid nodules, Reg treatment was associated with a marked nodule size reduction with complete disappearance in two of them without suspicious features of malignancy.

\section{Thyroid dysfunction and fatigue during Reg administration}

In Table 3 are reported age, sex, thyroid function tests and grading of fatigue in eleven patients who developed
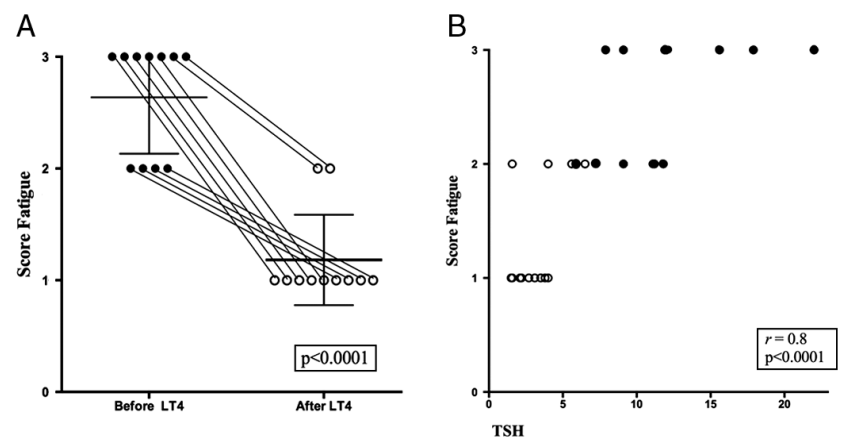

Figure 3

(A) Individual and mean \pm S.D. fatigue score before and after L-T4 therapy in the 11 hypothyroid patients submitted to L-T4 therapy ( $P<0.0001$ by Mann-Whitney test). (B) Correlation between fatigue score and serum TSH observed in all hypothyroid patients of before (solid circle) and after (open circle) L-T4 therapy.

clinical or subclinical hypothyroidism, before and after replacement L-T4 therapy. As shown in Fig. 3A, L-T4 treatment was associated with a marked decrease in median fatigue score $(P<0.0001$ by Mann-Withney test). Moreover, as shown in Fig. 3B, a highly significant direct correlation was found between serum TSH and fatigue score when data from untreated and treated patients were pooled together $(r=0.8, P<0.0001$ by linear correlation).

\section{Discussion}

This study is the first that prospectively analyzes thyroid function, thyroid autoimmunity and ultrasound findings in patients with mCRC during Reg treatment. About one half of patients developed mild subclinical hypothyroidism, and this prevalence is in agreement with that reported in the few retrospective studies in Reg-treated patients $(3,4,5)$. However, the prospective nature of the present investigation allowed to better characterize the onset of thyroid failure, which was somewhat earlier as compared to others TKIs (14). In particular, Reg-induced hypothyroidism was observed since the first month of therapy, whereas in a similar prospective study, sunitinib-induced hypothyroidism started after 3-4 months of treatment (15). The mechanisms involved in Reg-induced hypothyroidism have not been specifically investigated so far, but VEGF inhibition is considered the main mechanism common to all TKIs that cause thyroid failure (16). We recently provided evidence for a role of thyroid autoimmunity in 
the development of the most severe forms of Sunitinibinduced hypothyroidism (15). Similar to sunitinib, Reg administration was also associated with the novo appearance of TPOAb antibodies, further confirming that TKI treatment may be associated with triggering of thyroid autoimmunity. The appearance of TPOAb was observed in $2(8 \%)$ patients, a frequency lower than that reported in sunitinib-treated patients who developed TPOAb in about $30 \%$ of cases (15). It should be noted, however, that this difference can be at least partially due to the shorter follow-up available in the present study, in keeping with the low life expectation of Reg-treated mCRC patients; these preliminary data also suggest that each TKI could be characterized by its own different specific ability to activate the immune system and remains the matter of future investigations. Interestingly, the activation of the immune system could be related to the drug effectiveness, as suggested by the longer survival of sunitinb-treated patients with metastatic renal cancer who developed thyroid autoimmunity and more severe forms of hypothyroidism, which appears to represent a potential prognostic biomarker of better response (17), see below. Further studies are needed to clarify whether and to what extent this phenomenon is also present in Reg-treated patients. Moreover, the thyroid ultrasound evaluation showed an early and progressive reduction of thyroid volume with marked shrinkage or complete disappearance of thyroid nodules (when present) associated with a reduction of thyroid echogenicity, more evident in the two TPOAb-positive patients.

Taken together, these data suggest that the most important effects of Reg on thyroid (anti-angiogenic activity and exacerbation of thyroid autoimmunity) are similar to the effects exerted by sunitinib $(9,18,19,20$, 21) although the relative relevance is probably different.

Patients developing hypothyroidism had a highly significant longer survival when compared to euthyroid patients. This finding confirms several previous reports $(22,23)$, but the mechanism(s) underlying the "protective» effect of hypothyroidism is not known. The most common explanation for this phenomenon $(14,17$, 18 ) is that autoimmunity could represent a biomarker of oncological response. However, this mechanism remains highly hypothetical and far from being fully ascertained. Indeed, some authors suggested that hypothyroidism per se could be related to longer survival and therefore that L-T4 therapy might be contraindicated $(18,19,23)$. On the contrary, the results of the present study suggest that L-T4 treatment not only eliminated most of the fatigue symptoms, but also did not change the «protective» effect on survival in patients developing thyroid failure. Longer and controlled prospective studies will be needed to confirm these preliminary observations.

This study strongly suggests an important role of hypothyroidism in the pathogenesis of CRF. This concept is supported in one hand, by the direct correlation between serum TSH and fatigue grading and on the other hand, by the marked reduction of fatigue in hypothyroid patients treated with L-T4 at doses able to normalize serum TSH. Fatigue, defined by the Common Terminology Criteria for Adverse Events, version 4.03 (24), is the most frequent and the main AE that characterizes mCRC Regtreated patients; it is an undefined symptom related to disability to perform activities of daily living with a consequent psychological distress (6) and is one of the most difficult toxicity-related AE to manage $(25,26,27$, 28 ). For this reason, not infrequently, the presence of severe CRF leads to drug withdrawal. Interestingly, in the present study, the early recognition and treatment of hypothyroidism allowed the prosecution of Reg therapy in all patients due to rapid resolution of fatigue. We are aware that in the absence of a controlled design, this study does not allow a well-defined conclusion on the utility of L-T4 treatment of CRF. However, the persistence of the severe CRF observed in our hypothyroid patients before L-T4 administration would probably have led to Reg withdrawal, whereas the rapid improvement of fatigue after L-T4 administration allowed all patients to keep on Reg therapy.

In conclusion, these data indicate that Reg rapidly causes hypothyroidism in about $50 \%$ of patients and in a minority of them also triggers thyroid autoimmunity. Reg-induced hypothyroidism was strictly related to fatigue and easily reversed by L-T4 administration and associated to longer survival. Although further studies are needed to characterize longer-term effects of Reg on thyroid function/autoimmunity (also as biomarker of clinical response), these results suggest that prompt recognition of hypothyroidism in patients with severe fatigue prevent unnecessary Reg dose reduction or withdrawal. Finally, this study further strengths the concept that in patients submitted to TKI therapy management within multidisciplinary groups including oncologists and endocrinologists should be considered mandatory.

\section{Declaration of interest}

The authors declare that there is no conflict of interest that could be perceived as prejudicing the impartiality of this study. 
Funding

This work was partially supported by funds of the University of Cagliari (Contributo di Ateneo alla Ricerca) to S M.

\section{Author contribution statement}

F Pani and S Mariotti contributed to data analysis and interpretation, preparation of the manuscript. M Massidda, M Puzzoni, C Madeddu, E Massa and M Scartozzi contributed to data collection.

\section{Acknowledgments}

The authors sincerely thank Dr Giorgio Astara, from Medical Oncology Unit, Department of Medical Sciences and Public Health, University of Cagliari and Dr Grazia Pusole from Medical Oncology Unit, Department of Medical Sciences and Public Health, University of Cagliari for enrolling patients.

\section{References}

1 Wilhelm SM, Dumas J, Adnane L, Lynch M, Carter CA, Schütz G, Thierauch KH \& Zopf D. Regorafenib (BAY 73-4506): a new oral multikinase inhibitor of angiogenic, stromal and oncogenic receptor tyrosine kinases with potent preclinical antitumor activity. International Journal of Cancer 2011129 245-255. (doi:10.1002/ijc.25864)

2 Kircher SM, Nimeiri HS \& Benson Al B. Targeting angiogenesis in colorectal cancer. Cancer Journal 201622 182-189. (doi:10.1097/ PPO.0000000000000192)

3 Grothey A, Cutsem EV, Sobrero A, Siena S, Falcone A, Ychou M, Humblet $\mathrm{Y}$, Bouchè $\mathrm{O}$, Mineur L, Barone $\mathrm{C}$ et al. Regorafenib monotherapy for previously treated metastatic colorectal cancer (CORRECT): an international, multicenter, randomized, placebocontrolled, phase III trial. Lancet 2013381 303-312. (doi:10.1016/ S0140-6736(12)61900-X)

4 Demetri GD, Reichardt P, Kang YK, Blay JY, Rutkowski P, gelderblom $\mathrm{H}$, Hohenberger P, Leahy M, von Mehren M, Joensuu $\mathrm{H}$ et al. Efficacy and safety of regorafenib for advanced gastrointestinal stromal tumors after failure of imatinib and sunitinib (GRID): an international, multicenter, randomized, placebo-controlled, phase III trial. Lancet 2013381 295-302. (doi:10.1016/S0140-6736(12)61857-1)

5 Sugita K, Kawakami K, Yokokawa T, Mae Y, Toya W, Hagino A, Suzuki $\mathrm{K}$, Suenaga M, Mizunuma N, Yamaguchi T et al. Investigation of regorafenib-induced hypothyroidism in patients with metastatic colorectal cancer. Anticancer Research 201535 4059-4062.

6 Ryan JL, Carroll JK, Ryan EP, Mustian KM, Fiscella K \& Morrow GR. Mechanisms of cancer-related fatigue. Oncologist 200712 22-34. (doi:10.1634/theoncologist.12-S1-22)

7 Hofman M, Ryan JL, Figueroa CD, Moseley-Figueroa CD, Jean-Pierre P \& Morrow GR. Cancer-related fatigue: the scale of the problem. Oncologist 200712 4-10. (doi:10.1634/theoncologist.12-S1-4)

8 Santoni M, Conti A, Massari F, Arnaldi G, Iacovelli R, Rizzo M, De Giorgi U, Trementino L, Procopio G, Tortora G et al. Treatmentrelated fatigue with sorafenib, sunitinib and pazopanib in patients with advanced solid tumors: an up-to-date review and meta-analysis of clinical trials. International Journal of Cancer 2015136 1-10. (doi:10.1002/ijc.28715)

9 Torino F, Barnabei A, Paragliola R, Baldelli R, Appetecchia M \& Corsello SM. Thyroid dysfunction as an unintended side effect of anticancer drugs. Thyroid 201311 1345-1366. (doi:10.1089/ thy.2013.0241)

10 Oken MM, Creech RH, Tormey DC, Horton J, Davis TE, Mc Fadden ET \& Carbone PP. Toxicity and response criteria of the Eastern
Cooperative Oncology Group. American Journal of Clinical Oncology 19825 649-655. (doi:10.1097/00000421-198212000-00014)

11 Ruan M, Shen Y, Chen L \& Li M. RECIST 1.1 and serum thyroglobulin measurements in the evaluation of responses to sorafenib in patients with radioactive iodine-refractory differentiated thyroid carcinoma. Oncology Letters 20136 480-486. (doi:10.3892/ol.2013.1424)

12 Jonklaas J, Bianco AC, Bauer AJ, Burman KD, Cappola AR, Celi FS, Cooper DS, Kim BW, Peeters RP, Rosenthal MS et al. Guidelines for the treatment of hypothyroidism: prepared by the American Thyroid Association task force on thyroid hormone replacement. Thyroid 2014 24 1670-1751. (doi:10.1089/thy.2014.0028)

13 Berghout A, Wiersinga WM, Smits NJ \& Touber JL. The value of thyroid volume measured by ultrasonography in the diagnosis of goiter. Clinical Endocrinology 198828 409-414. (doi:10.1111/j.1365-2265.1988.tb03672.x)

14 Bianchi L, Rossi L, Tomao F, Papa A, Zoratto F \& Tomao S. Thyroid dysfunction and tyrosine kinase inhibitors in renal cell carcinoma. Endocrine-Related Cancer 201320 233-245. (doi:10.1530/ERC-130201)

15 Pani F, Atzori F, Baghino G, Boi F, Tanca L, Ionta MT \& Mariotti S. Thyroid dysfunction in patients with metastatic carcinoma treated with sunitinib: is thyroid autoimmunity involved? Thyroid 201511 1255-1261. (doi:10.1089/thy.2015.0170)

16 Makita N \& Liri T 2013 Tyrosine kinase inhibitor-induced thyroid disorders: a review and hypothesis. Thyroid 23 151-159. (doi:10.1089/ thy.2012.0456)

17 Pani F, Atzori F, Baghino G, Boi F, Ionta MT, Tanca L, Scartozzi M \& Mariotti S. Hypothyroidism and thyroid autoimmunity as a prognostic biomarker of better response in metastatic cancer longterm survivors treated with sunitinib. Thyroid 20169 1336-1337. (doi:10.1089/thy.2016.0159)

18 Illouz F, Braun D, Briet C, Schweizer U \& Rodien P. Endocrine side-effects of anti-cancer drugs: thyroid effects of tyrosine kinase inhibitors. European Journal of Endocrinology 2014171 91-99. (doi:10.1530/EJE-14-0198)

19 Makita N, Miyakawa M, Fujita T \& Iiri T. Sunitinib induces hypothyroidism with a markedly reduced vascularity. Thyroid 201020 323-326. (doi:10.1089/thy.2009.0414)

20 Kappers MH, van Esch JH, Smedts FM, de Krijger RR, Eechoute K, Mathijssen RH, Sleijfer S, Leijten F, Danser AH, van den Meiracker $\mathrm{AH}$ et al. Sunitinib-induced hypothyroidism is due to induction of type 3 deiodinase activity and thyroidal capillary regression. Journal of Clinical Endocrinology and Metabolism 201196 3087-3094. (doi:10.1210/jc.2011-1172)

21 Grossmann M, Premaratne E, Desai J \& Davis ID. Thyrotoxicosis during sunitinib-treatment for renal cell carcinoma. Clinical Endocrinology 200869 669-672. (doi:10.1111/j.13652265.2008.03253.x)

22 Sabatier R, Eymard JC, Walz J, Deville JL, Narbonne H, Boher JM, Salem N, Marcy M, Brunelle S, Viens P et al. Could thyroid dysfunction influence outcome in sunitinib-treated metastatic renal cell carcinoma? Annals of Oncology 201423 714-721. (doi:10.1093/ annonc/mdr275)

23 Kust D, Prpić M, Murgić J, Jazvić M, Jakšić B, Krilić D, Bolanča A \& Kusić Z. Hypothyroidism as a predictive clinical marker of better treatment response to sunitinib therapy. Anticancer Research 201434 3177-3184

24 Common Terminology Criteria for Adverse Events (CTCAE) version 4.03, June 2010 NIC: General disorders and administration site conditions: 22 .

25 Li J, Qin S, Xu R, Yau TC, Ma B, Pan H, Xu J, Bai Y, Chi Y, Wang $\mathrm{L}$ et al. Regorafenib plus best supportive care versus placebo plus best supportive care in Asian patients with previously treated metastatic colorectal cancer (CONCUR): a randomised, double-blind, placebo-controlled, phase 3 trial. Lancet Oncology 201516 619-629. (doi:10.1016/S1470-2045(15)70156-7) 
26 Hofheinz RD, Arnold D, Kubicka S, Prasnikar N \& Vogel A. Improving patient outcomes with regorafenib for metastatic colorectal cancerpatients selection, dosing, patient education, prophylaxis, and management of adverse events. Oncology Research and Treatment 2015 38 300-308. (doi:10.1159/000382067)

27 Calcagno F, Lenoble S, Lakkis Z, Nguyen T, Limat S, Borg C, Jary M, Kim $S$ \& Nerich V. Efficacy, safety and cost of regorafenib in patients with metastatic colorectal cancer in French clinical practice. Clinical Medicine Insights: Oncology 201610 59-66.

28 Sastre J, Argilès G, Benavides M, Feliù J, Garcìa-Alfonso P, GarcìaCarbonero R, Gràvalos C, Guillèn-Ponce C, Martìnez-Villacampa M $\&$ Pericay C. Clinical management of regorafenib in the treatment of patients with advanced colorectal cancer. Clinical and Translational Oncology 201411 942-953. (doi:10.1007/s12094-014-1212-8)

Received 20 March 2017

Revised version received 24 April 2017

Accepted 2 May 2017 